Check for updates

Cite this: RSC Adv., 2020, 10, 20809

\title{
The broad-spectrum and ultra-sensitive detection of zeranol and its analogues by an enzyme-linked immunosorbent assay in cattle origin samples
}

\author{
Mengqi Yin, (D) ab Xiaofei Hu, (D) ${ }^{b}$ Yaning Sun, ${ }^{b}$ Yunrui Xing, ${ }^{b}$ Shujun Chai, ${ }^{b}$ \\ Guangxu Xing, ${ }^{\mathrm{b}}$ Yanyan Yang, ${ }^{\mathrm{b}}$ Man Teng, ${ }^{\mathrm{b}}$ Qingmei Li, ${ }^{\mathrm{b}}$ Yao Wang, ${ }^{\mathrm{c}}$ Ruiguang Deng ${ }^{\mathrm{b}}$ \\ and Gaiping Zhang*abd
}

Zeranol ( $\alpha$-zearalanol) has been used as a growth promoter in livestock since 1969 in some non-EU countries; the residues of zeranol and its five analogues in animal origin foods may endanger human health due to their strong estrogenic and anabolic activities. Therefore, it is urgent to establish simple, rapid, real-time, broad-spectrum and high-sensitivity detection methods for the residues of zeranol and its analogues. In this study, an ultrasensitive indirect-competition enzyme-linked immunosorbent assay (ic-ELISA) was established for the rapid multi-residue detection of zeranol and its five analogues in cattle origin samples, which was based on a broad-spectrum monoclonal antibody (mAb) that specifically bound to zeranol and its analogues with high sensitivity. The half maximal inhibitory concentration $\left(I C_{50}\right)$ values for zeranol, $\beta$-zearalanol, zearalanone, $\alpha$-zearalenol, $\beta$-zearalenol, and zearalenone were 0.103 , $0.080,0.161,0.177,0.254$, and $0.194 \mathrm{ng} \mathrm{mL}^{-1}$, respectively, the recovery rates of cattle origin samples spiked with zeranol ranged from $79.2-104.2 \%$, and the coefficient of variation (CV) values were less than 11.4\%. Excellent correlation $\left(R^{2}=0.9845\right)$ was obtained between the results of HPLC-MS/MS and icELISA. In conclusion, the developed ic-ELISA could be employed as an ultrasensitive and broadspectrum detection method for monitoring trace ZEN residues in cattle origin foods.

Received 31st March 2020

Accepted 11th May 2020

DOI: 10.1039/dOra02936j

rsc.li/rsc-advances
$(\beta$-ZEL) and zearalenone (ZEA), are also similar in configuration to $\mathrm{E} 2$ and also possess these activities (Fig. 1). ${ }^{9}$ The in vivo metabolism of the six analogues in fungi, plants and animals could lead to more than one compound residue in related products. Moreover, these compounds are tolerant of heat and milling, and are difficult to inactivate and remove during

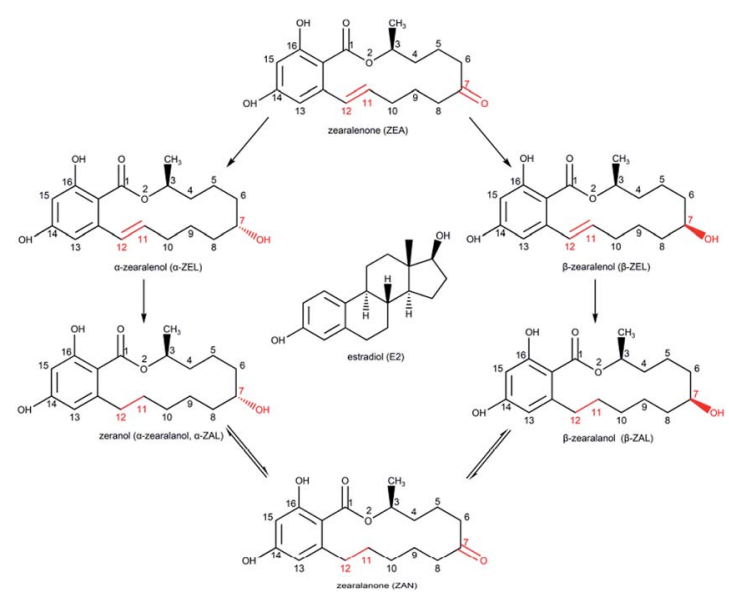

Fig. 1 The chemical structures of ZENs and E2.
${ }^{a}$ School of Food Science and Technology, Jiangnan University, Wuxi, Jiangsu 214122, China. E-mail: zhanggaip@126.com

${ }^{b}$ Key Laboratory of Animal Immunology, Henan Academy of Agricultural Sciences, Zhengzhou 450002, China

'College of Food and Bioengineering, Henan University of Science and Technology, Luoyang 471023, China

${ }^{d}$ College of Animal Science and Veterinary Medicine, Henan Agricultural University, Zhengzhou 450002, China is the major female sex hormone, thus it possesses estrogenic and anabolic activities. Therefore, zeranol has been approved conversion in livestock in some non-EU countries since 1969. ${ }^{2,3}$ However, various studies have demonstrated that zeranol could disorders, developmental problems, nephrotoxicity, hepatotoxicity and a higher risk of breast cancer. ${ }^{4-8}$ In addition, the homologous analogues of zeranol, including $\beta$-zearalanol $(\beta$-ZAL), zearalanone (ZAN), $\alpha$-zearalenol $(\alpha$-ZEL), $\beta$-zearalenol 
processing or cooking. ${ }^{10}$ Thus, zeranol and its analogues, named as ZENs in this study, could be classified as estrogenic food contaminants in the human diet. To protect consumers from these contaminants, most international organizations and countries have set regulations for permissible levels of zeranol in cattle origin foods, ${ }^{11,12}$ while zeranol is banned for use in livestock and must not be detected in cattle origin foods in the $\mathrm{EU}^{\mathbf{1 3}}$ and China. ${ }^{\mathbf{1 4}}$ However, it is not enough to just detect one ZEN in one sample at one time: a satisfactory detection method should simultaneously detect all ZENs or the total level of ZENs in one sample at one time with sufficient sensitivity and specificity.

In recent decades, numerous detection methods for zeranol residues in foods have been established, such as gas chromatography-mass spectrometry $\quad$ (GC-MS), ${ }^{15}$ liquid chromatography-tandem mass spectrometry (LC-MS/MS), ${ }^{\mathbf{1 6}}$ high performance liquid chromatography-tandem mass spectrometry (HPLC-MS) ${ }^{17}$ and ultra-high performance liquid chromatography-tandem mass spectrometry (UHPLC-MS/MS). ${ }^{6}$ These methods have the advantages of high sensitivity and reliability, but they demand expensive precision instruments, complicated sample preparation, experienced professional operators and highly regimented experimental environments. Recently, immunoassay methods, based on the interaction between antibodies and target specific molecules, have been extensively used, such as the radio immunoassay (RIA), ${ }^{18}$ chemiluminescence immunoassay (CLIA), ${ }^{19}$ electrochemical immunosensing (ECIS), ${ }^{20}$ time-resolved fluorescence immunoassay (TR-FIA), ${ }^{21}$ and enzyme-linked immunosorbent assay (ELISA). ${ }^{22}$ Compared with the methods involving precision instruments, immunoassay methods are more cost-efficient and easy-to-use with sufficient sensitivity and specificity, which can satisfy the requirements for rapid detection. Among these methods, ELISA is the most commonly developed immunoassay method for detecting mycotoxins, prohibited additives, and veterinary drugs.

In immunoassays, the antibody is the first critical factor and will directly affect the performance of the developed ELISA method. To detect a class of compounds such as ZENs, the antibody should have broad specificity, similar sensitivity and uniform affinity to all the analytes. Hence, the aim of this study was to prepare a broad-specific monoclonal antibody (mAb) for ZENs with similar sensitivity and uniform affinity, and then develop an ELISA method for multi-residue detection of zeranol and its analogues in cattle origin samples. The developed ELISA should detect the total level of ZENs and have the advantages of simple sample preparation, insusceptibility to matrix effects, and sufficient sensitivity and specificity.

\section{Materials and methods}

\subsection{Chemicals, materials, and apparatus}

$\alpha$-Zearalanol ( $\alpha$-ZAL, zeranol), $\beta$-zearalanol ( $\beta$-ZAL), zearalanone (ZAN), $\alpha$-zearalenol ( $\alpha$-ZEL), $\beta$-zearalenol ( $\beta$-ZEL), zearalenone (ZEA), aflatoxin B1 (AFB1), deoxynivalenol (DON), fumonisin B1 (FB1), ochratoxin A (OTA), patulin (PAT), T-2 toxin, $O$-(carboxymethyl) hydroxylamine hemihydrochloride (CMO), 1-(3- (dimethylamino)propyl)-3-ethylcarbodiimide hydrochloride (EDC), $\mathrm{N}$-hydroxysuccinimide (NHS), Freund's complete adjuvant (FCA), Freund's incomplete adjuvant (FIA), a mouse monoclonal antibody isotyping kit and $\beta$-glucuronidase/ sulfatase were purchased from Sigma-Aldrich (St. Louis, USA). Trenbolone acetate (TBA), melengestrol acetate (MLGA) and progesterone (P4) were purchased from Dr Ehrenstorfer $\mathrm{GmbH}$ (Augsburg, Germany). Bovine serum albumin (BSA) and ovalbumin (OVA) were purchased from BDH (VWR International Ltd., USA). RPMI-1640 medium was purchased from Solarbio (Beijing, China). Fetal bovine serum (FBS), cell culture plastics and a goat anti-mouse IgG antibody (whole molecule) were obtained from Thermo Fisher Scientific (Waltham, USA). Ninety-six-well high adsorption ELISA plates were purchased from GSBIO (Wuxi, China). The other reagents and solvents were of analytical grade or higher.

Eight-week-old female BALB/c mice were obtained from the Laboratory Animal Centre (Zhengzhou University, China). Animal immunizations were performed at the Henan Key Laboratory of Animal Immunology (Zhengzhou, China). All animals were raised and treated according to the principles of Institutional Animal Care and Use Committees of the Henan Academy of Agricultural Sciences.

Murine myeloma SP2/0 cells were provided by Dr Howard, Institute for Animal Health, UK, and maintained in RPMI-1640 medium supplemented with $10 \%$ (v/v) FBS.

A microplate reader 550 was purchased from Bio-Rad (Richmond, USA). An ultrapure water purification system was purchased from Millipore (Bedford, USA).

\subsection{Production of monoclonal antibodies}

The synthesis of hapten-protein conjugates was performed according to previous studies with modifications, as described in Fig. $2 .{ }^{23}$ Five milligrams of ZAN and $10 \mathrm{mg}$ of CMO were dissolved in $5 \mathrm{~mL}$ of pyridine, the mixture was stirred at $100{ }^{\circ} \mathrm{C}$ for $6 \mathrm{~h}$ and dried under vacuum, and the residue was redissolved in $5 \mathrm{~mL}$ of distilled water (the $\mathrm{pH}$ was adjusted to 8 with $2 \mathrm{M}$ sodium bicarbonate buffer). Unmodified ZAN was extracted from the aqueous phase with $5 \mathrm{~mL}$ of methylbenzene three times. Then, the aqueous phase was extracted with $5 \mathrm{~mL}$ of ethyl acetate three times. Subsequently, the ethyl acetate phase was dried under vacuum, and the residue was the oxime ZAN-7-CMO. ZAN-7-CMO was dissolved in $1 \mathrm{~mL}$ of $N, N$ -

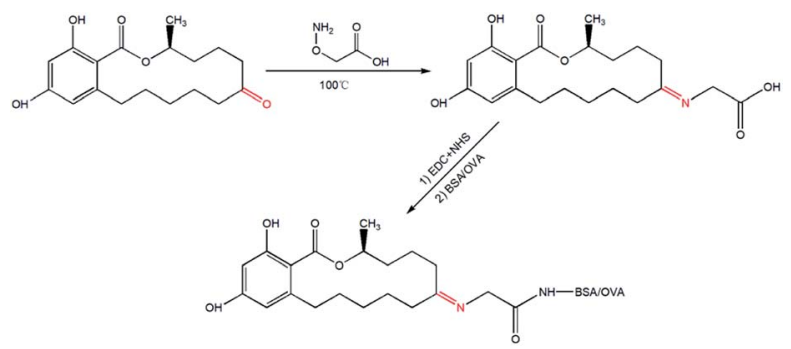

Fig. 2 The synthetic scheme used to produce the hapten-protein conjugates. 
dimethylformamide and transferred into a $10 \mathrm{~mL}$ glass bottle with $5 \mathrm{mg}$ of EDC and $3 \mathrm{mg}$ of NHS. The mixture ZAN-7-CMO/ EDC/NHS was stirred gently at $4{ }^{\circ} \mathrm{C}$ for $12 \mathrm{~h}$. Next, $13 \mathrm{mg}$ of BSA or $9 \mathrm{mg}$ of OVA was dissolved in $2 \mathrm{~mL}$ of $0.01 \mathrm{M}$ phosphatebuffered saline (PBS, pH 7.4) and the mixture ZAN-7-CMO/EDC/ NHS was added dropwise under slight stirring. The reaction mixture ZAN-7-CMO/EDC/NHS/protein was stirred gently at $4{ }^{\circ} \mathrm{C}$ for $12 \mathrm{~h}$. Finally, the conjugates ZAN-BSA and ZAN-OVA were dialyzed in $0.01 \mathrm{M}$ PBS at $4{ }^{\circ} \mathrm{C}$ for $72 \mathrm{~h}$ and stored at $-20{ }^{\circ} \mathrm{C}$.

Each BALB/c mouse was subcutaneously immunized with 50 $\mu \mathrm{g}$ of ZAN-BSA. The first injection was performed with $100 \mu \mathrm{L}$ of the immunogen which was emulsified with $100 \mu \mathrm{L}$ of FCA, and the other three booster injections were carried out at intervals of 3 weeks with $100 \mu \mathrm{L}$ of immunogen emulsified in $100 \mu \mathrm{L}$ of FIA. Antisera were collected on the 14th day after the fourth immunization to investigate the sensitivity and specificity of the anti-zeranol antibody. The titre of the anti-zeranol antibody and the recognition property of the ZENs were tested by indirect ELISA (i-ELISA) and indirect competitive ELISA (ic-ELISA), respectively. The mouse that could produce antibodies with the highest anti-zeranol activity was selected for cell fusion. ${ }^{24}$ Ten days later, the cell fusion supernatants were screened by icELISA. Positive hybridoma cells were subcloned to obtain monoclonal cells by limiting dilution and then screened by icELISA. Monoclonal cells with the highest titre and best specificity were used to produce ascitic fluids in BALB/c mice after pretreatment with paraffin, and anti-zeranol mAbs were obtained by purifying the ascites by the ammonium sulfate method.

\subsection{Protocol of i-ELISA and ic-ELISA}

ELISA plates were added to $100 \mu \mathrm{L}$ per well of ZAN-OVA in $0.05 \mathrm{M}$ carbonate-bicarbonate buffer (CBS, pH 9.6), and incubated at $37^{\circ} \mathrm{C}$ for $2 \mathrm{~h}$; after washing with $0.01 \mathrm{M}$ PBS with tween$20(0.05 \%, \mathrm{v} / \mathrm{v})(\mathrm{PBST}, \mathrm{pH} 7.4)$, the plates were blocked by $270 \mu \mathrm{L}$ per well of skimmed milk $(5 \%, \mathrm{w} / \mathrm{v})$ in PBST and incubated at $37^{\circ} \mathrm{C}$ for $2 \mathrm{~h}$; after washing, the prepared plates were stored at $4{ }^{\circ} \mathrm{C}$.

For i-ELISA, firstly, $100 \mu \mathrm{L}$ per well of antiserum in $5 \%$ skimmed milk was added to the prepared plate, followed by incubation at $37^{\circ} \mathrm{C}$ for $15 \mathrm{~min}$; after washing, $100 \mu \mathrm{L}$ per well of HRP-IgG in $5 \%$ skimmed milk ( $1: 1000$, v/v) was added, followed by incubation at $37^{\circ} \mathrm{C}$ for $30 \mathrm{~min}$; then, after washing and drying, $100 \mu \mathrm{L}$ per well of the TMB substrate solution was added, followed by incubation at $37{ }^{\circ} \mathrm{C}$ for $10 \mathrm{~min}$; finally, the reaction was terminated by adding $100 \mu \mathrm{L}$ per well of $2 \mathrm{M}$ sulfuric acid. The optical densities (OD) were determined at $450 \mathrm{~nm}$ by the microplate reader. For ic-ELISA, the detection procedures were similar to the above, except that $100 \mu \mathrm{L}$ per well of standard solution was added simultaneously in the first step.

\subsection{Optimization and establishment of the ic-ELISA method}

The optimal ic-ELISA working conditions were determined as the optimal working concentration of the coating antigen with the optimal dilution of mAb. To determine the optimal ic-ELISA conditions, different concentrations of ZAN-OVA $(0.1875$ to $3 \mu \mathrm{g}$
$\left.\mathrm{mL}^{-1}\right)$ with a series of dilutions of anti-zeranol mAb (1:1000 to $1: 64000, \mathrm{v} / \mathrm{v}$ ) were screened by checkerboard titration.

Under the optimal working conditions, the assay buffer was investigated using ic-ELISA. In this study, a standard solution of zeranol ranging from 0.0098 to $2.5 \mathrm{ng} \mathrm{mL}^{-1}$ was prepared in PBS buffers containing different amounts of methanol, then the standard curve was obtained by importing the measured data into GraphPad Prism software to fit to a four-parameter equation.

The developed ic-ELISA was used to investigate the specificity of anti-zeranol mAb against the analogues of zeranol, other mycotoxins and hormonal growth promoters, including $\beta$ ZAL, $\alpha$-ZEL, $\beta$-ZEL, ZAN, ZEA, AFB1, FB1, DON, OTA, PAT, T-2 toxin, TBA, MLGA and P4. The specificity of the anti-zeranol $\mathrm{mAb}$ against the analytes was evaluated by the value of the half maximal inhibitory concentration $\left(\mathrm{IC}_{50}\right)$, which was the concentration that could inhibit the specific molecular recognition between the coating antigen and antibody by half, acquired from a four-parameter logistic fit of the standard points. The values of cross-reactivity (CR) were calculated according to the following equation:

$$
\mathrm{CR}(\%)=\frac{\mathrm{IC}_{50} \text { of zeranol }}{\mathrm{IC}_{50} \text { of other compounds }} \times 100 \% \text {. }
$$

\subsection{Preparation of the cattle origin sample}

Milk, skim milk powder and bovine muscle were purchased from formal markets. Bovine liver and kidney were collected from controlled slaughterhouses. Bovine urine samples were collected from the cattle farm affiliated to the Henan Academy of Agricultural Sciences. All the samples were confirmed as negative (no ZENs detected) by HPLC.

Milk, skim milk powder and urine. Five microliters of each liquid samples were centrifuged at $12000 \mathrm{rpm}$ for $20 \mathrm{~min}$ at $4{ }^{\circ} \mathrm{C}$ to form sediment particulate matter or a lipid layer. $2.5 \mathrm{~g}$ of skim milk powder in $5 \mathrm{~mL}$ of distilled water was processed similarly to the above. $1 \mathrm{~mL}$ of the supernatants or middle layers were taken and de-conjugated by incubation at $37{ }^{\circ} \mathrm{C}$ for $3 \mathrm{~h}$ with $50 \mu \mathrm{L}$ of $\beta$-glucuronidase/sulfatase. Finally, the samples were diluted with the methanol-PBS assay buffer before analysis with ic-ELISA.

Muscle, liver and kidney. After removing the fat and fascia tissue, bovine muscle, liver and kidney samples were finely minced, then $2 \mathrm{~g}$ of the sample and $4 \mathrm{~mL}$ of distilled water containing $16 \mu \mathrm{L}$ of $\beta$-glucuronidase/sulfatase were added together into a $50 \mathrm{~mL}$ polypropylene centrifuge tube. After incubating at $37^{\circ} \mathrm{C}$ for $3 \mathrm{~h}, 16 \mathrm{~mL}$ of methanol was added. After being shaken vigorously for $10 \mathrm{~min}$, the sample mixture was centrifuged at $5000 \mathrm{rpm}$ for $10 \mathrm{~min}$. Subsequently, the supernatant was collected and diluted with $0.1 \mathrm{M}$ PBS before analysis with ic-ELISA.

In the livestock breeding process approximately $35 \%$ of the absorbed zeranol was excreted via the urinary system, and if animals were fed with ZEN-contaminated feedstuff, about 33\% of the ZENs (the sum of ZEA, the metabolites and the respective isomers) could be excreted as above. Therefore, in this study, 
bovine urine was selected as one sample to evaluate the developed immunoassay, aiming to monitor ZEN contamination.

\subsection{Validation of the ic-ELISA method}

The ic-ELISA was validated on the basis of several parameters, including the limit of detection (LOD), the limit of quantification (LOQ), accuracy, precision and reliability. The values of LOD and LOQ were determined with 20 blank negative samples which were prepared based on the results of the matrix effects analysis. LOD was the concentration corresponding to the sum of the mean value of 20 blank samples plus three times the standard deviation (SD) and LOQ was the concentration corresponding to the sum of the mean value of 20 blank samples plus ten times the SD.

The accuracy and precision were evaluated by the recovery rate (RV) and the coefficient of variation (CV), respectively. Six blank samples were prepared and spiked with concentrations corresponding to 1, 2 and 5 times the LOQ values in triplicate, and the RVs were calculated according to the following equation:

$$
\mathrm{RVs}=\frac{\text { measured concentration }}{\text { spiked concentration }} \times 100 \%
$$

The reliability was determined from the correlation between the ic-ELISA and HPLC-MS/MS results. Four negative milk samples were spiked with different concentrations of zeranol and then equally divided into two groups. One group was analysed by ic-ELISA, and the other group was analysed by HPLCMS/MS.

\section{Results and discussion}

\subsection{Antigen selection}

Zeranol, as well as the five other ZENs, is a typical hapten; haptens are very small molecules $(\mathrm{MW}<1 \mathrm{kDa})$ that can elicit an immune response and stimulate the production of antibodies only when conjugated to carrier proteins. The structures of ZENs are almost identical, except for differences at positions 7 and 11 on the ring. Suitable carrier proteins and the most preferable synthesis pathways were the most influential factors affecting hapten conjugation, and these factors relate to the production and sensitivity of the antibody. The two most common carrier proteins, BSA and OVA, were employed for hapten conjugation in this study. To select a preferable conjugation method, the functional groups of ZENs and the carrier proteins must be identified.

In preliminary tests of this work, five synthesis pathways were employed which involved two positions on the ring of the
ZENs. In the first pathway, position 16 of $\alpha$-ZAL was modified to produce $\alpha$-ZAL-16-carboxypropyl ether; then, the ether was coupled with proteins in a mixed anhydride reaction. The second pathway was similar to the first; the position-7 hydroxyl group of $\alpha$-ZAL was modified by insertion of an alkyl chain, resulting in $\alpha$-ZAL-7-hemisuccinate, which was then conjugated to proteins by the active ester method. The principles of the third through fifth pathways were similar, position 7 of $\alpha$-ZAL, ZAN and ZEA was modified by inserting a carboxyl group and then coupled with the carrier proteins BSA and OVA by the active ester method.

According to the results of the preliminary tests, the antiserum of antigen ZAN-BSA (ZAN-7-CMO-BSA, the fourth pathway) had the highest specificity towards zeranol, and the subsequent results shown in Table 2 indicate that the mAb obtained from antigen ZAN-BSA exhibited excellent broadspectrum specificity for all ZENs.

\subsection{Optimization and specificity of the ic-ELISA method}

Immunoassay methods mainly rely on the effective combination of an antibody with an antigen, the sensitivity of the mAb is a decisive factor. In summary, a mAb for zeranol and other ZENs with broad specificity and high sensitivity is necessary to meet the requirements for the immunoassay. Fortunately, in this study, we obtained a more sensitive and broad-spectrum antizeranol $\mathrm{mAb}$ than other recently reported mAbs for ZENs. ${ }^{19,21,22,25}$ Therefore an ultrasensitive ic-ELISA can be established for the determination of zeranol and its analogues in one sample at one time.

The working concentrations of the coating antigen and mAb are decisive factors concerning the specificity and sensitivity of the ic-ELISA. For $\mathrm{IC}_{50}$, different concentration combinations of the coating antigen and mAb yield different results. In this assay, $\mathrm{IC}_{50}$ values were used to determine the optimal working concentrations of the coating antigen and mAbs by checkerboard titration. As shown in Table 1, the optimal working conditions were determined as $0.25 \mu \mathrm{g} \mathrm{mL}^{-1}$ of ZAN-OVA and a $1: 2 \times 10^{4} \mathrm{mAb}$ dilution, and remained constant after that.

Methanol is one of the most commonly used organic solvent for dissolving the standards or extracting and purifying the biological samples. However, the activity of the antibody could be affected by the amount of organic solvents in the sample. In order to minimize or eliminate the nonspecific interferences, the standard solution of zeranol was diluted with buffers containing different amount of methanol in PBS, and then analysed by ic-ELISA. As shown in Fig. 3a and b, with an increase of the methanol content in PBS, the $B_{0}$ values and the $\mathrm{IC}_{50}$ values of $\alpha$ ZAL were both increased. It is probably because the methanol

Table 1 Optimization of ic-ELISA via checkerboard titration

\begin{tabular}{|c|c|c|c|c|c|c|}
\hline $\begin{array}{l}\text { Concentration of coating antigen ( } \mu \mathrm{g} \\
\left.\mathrm{mL}^{-1}\right)\end{array}$ & 3 & 1.5 & 0.75 & 0.375 & 0.25 & 0.1875 \\
\hline Dilution of mAb $(1: X)$ & $2.7 \times 10^{4}$ & $2.5 \times 10^{4}$ & $2.4 \times 10^{4}$ & $2.2 \times 10^{4}$ & $2 \times 10^{4}$ & $1.6 \times 10^{4}$ \\
\hline $\mathrm{IC}_{50}\left(\mathrm{ng} \mathrm{mL} \mathrm{m}^{-1}\right)$ & 0.134 & 0.124 & 0.101 & 0.096 & 0.086 & 0.091 \\
\hline
\end{tabular}



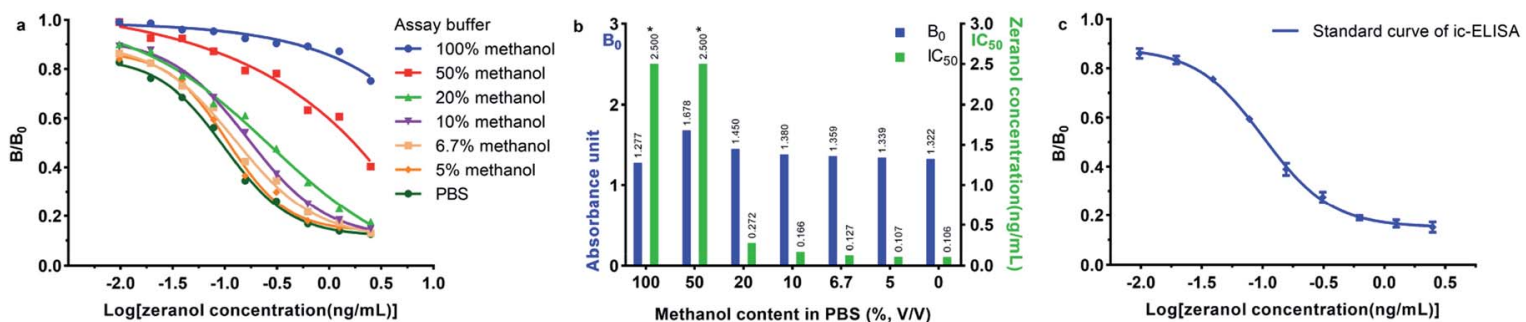

Fig. 3 The influence of the methanol content in PBS (v/v) used as the assay buffer in ic-ELISA against zeranol (a and b) and the standard curve of ic-ELISA for zeranol in $5 \%$ methanol (c); * the $\mathrm{IC}_{50}$ value was $>2.5 \mathrm{ng} \mathrm{mL}^{-1}$.

Table 2 Cross-reactivity of mAb with ZENs, other mycotoxins and growth promoters

\begin{tabular}{|c|c|c|c|c|c|c|c|c|}
\hline ZENs & $\mathrm{IC}_{50}\left(\mathrm{ng} \mathrm{mL} \mathrm{m}^{-1}\right)$ & CR $(\%)$ & Other mycotoxins & $\mathrm{IC}_{50}\left(\mathrm{ng} \mathrm{mL} \mathrm{m}^{-1}\right)$ & CR $(\%)$ & $\begin{array}{l}\text { Other growth } \\
\text { promoters }\end{array}$ & $\mathrm{IC}_{50}\left(\mathrm{ng} \mathrm{mL}^{-1}\right)$ & CR $(\%)$ \\
\hline$\alpha-\mathrm{ZAL}$ & 0.103 & 100.0 & AFB1 & $>1000$ & $<0.1$ & TBA & $>1000$ & $<0.1$ \\
\hline ZAN & 0.161 & 64.0 & FB1 & $>1000$ & $<0.1$ & $\mathrm{P} 4$ & $>1000$ & $<0.1$ \\
\hline$\alpha-Z E L$ & 0.177 & 58.2 & OTA & $>1000$ & $<0.1$ & & & \\
\hline$\beta$-ZEL & 0.254 & 40.6 & PAT & $>1000$ & $<0.1$ & & & \\
\hline
\end{tabular}

might increase the non-specific protein adsorption but decrease the affinity of the anti-zeranol mAb for free zeranol. Specifically, when the methanol content in PBS was lower than $6.7 \%$, the activity of the antibody and the sensitivity of ic-ELISA were not changed. Therefore, in this assay, $5 \%$ of methanol was selected.

Under the optimum conditions, $5 \%$ of methanol in PBS buffer was used as the solvent of the standard solution and the extraction reagent of the real sample. As shown in Fig. 3c, the standard curve was obtained within 0.0098 to $2.5 \mathrm{ng} \mathrm{mL}^{-1}$, and the $B$ and $B_{0}$ represented the OD value $(450 \mathrm{~nm})$ of zeranol at different concentrations and at $0 \mathrm{ng} \mathrm{mL} \mathrm{mL}^{-1}$. The four-parameter equation was $Y=$ bottom $+\frac{\text { top-bottom }}{1+10^{\left[\left(\log \mathrm{IC}_{50}-X\right) \times \text { hill slope }\right]}}$, where bottom $=0.1509$, top $=0.8829, \log \mathrm{IC}_{50}=-0.9885$, hill slope $=$ -1.560 , and $R^{2}=0.9970$. As shown in Table 2 , the $\mathrm{IC}_{50}$ value for zeranol was $0.103 \mathrm{ng} \mathrm{mL}^{-1}$, and those for $\beta$-ZAL, ZAN, $\alpha$-ZEL, $\beta$ ZEL and ZEA were $0.080 \mathrm{ng} \mathrm{mL}^{-1}, 0.161 \mathrm{ng} \mathrm{mL}^{-1}, 0.177 \mathrm{ng}$ $\mathrm{mL}^{-1}, 0.254 \mathrm{ng} \mathrm{mL}^{-1}$ and $0.194 \mathrm{ng} \mathrm{mL}^{-1}$, respectively, with the
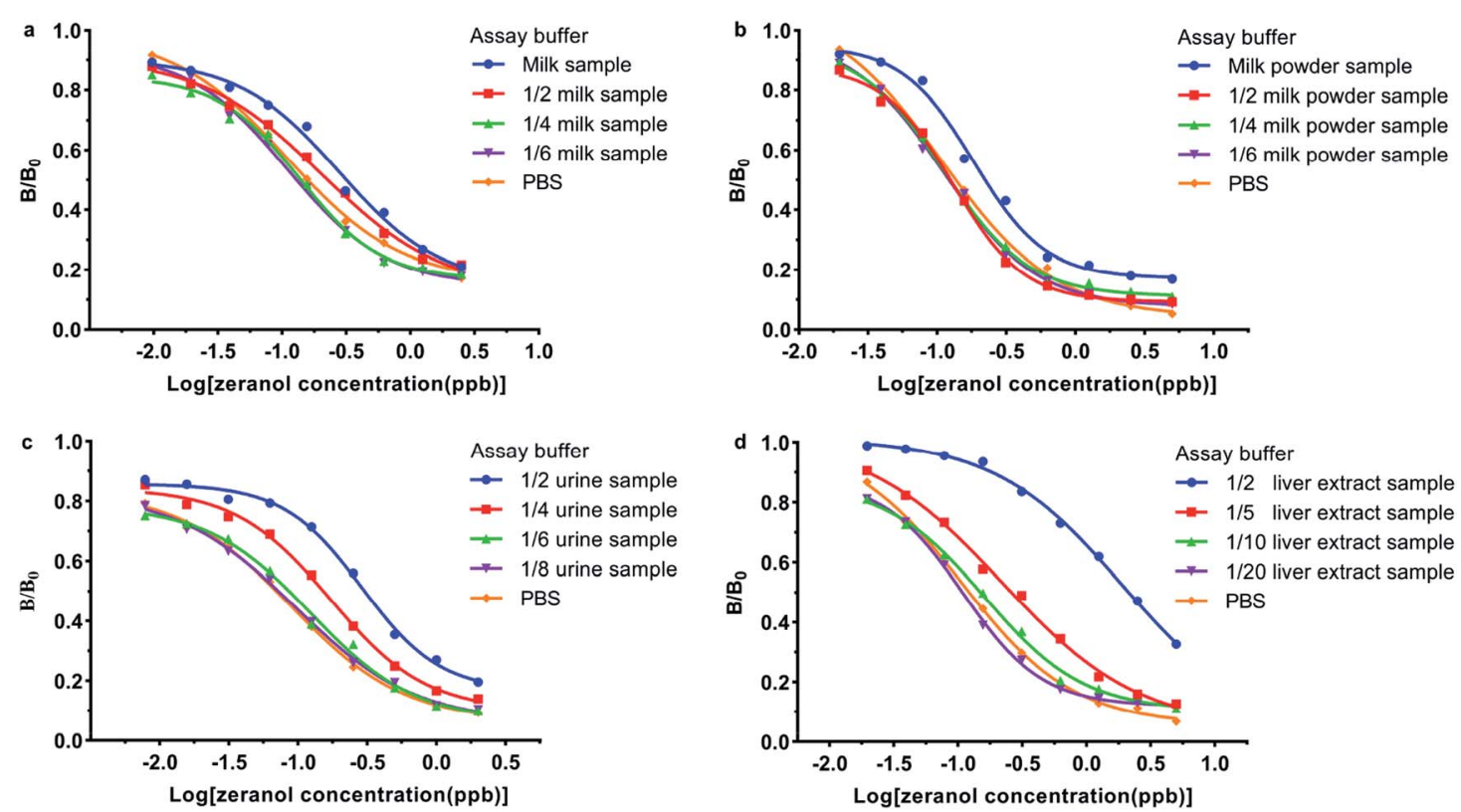

Fig. 4 The evaluation and elimination of the matrix effects in a (a) milk sample, (b) skim milk powder sample, (c) urine sample, and (d) liver extract sample. 

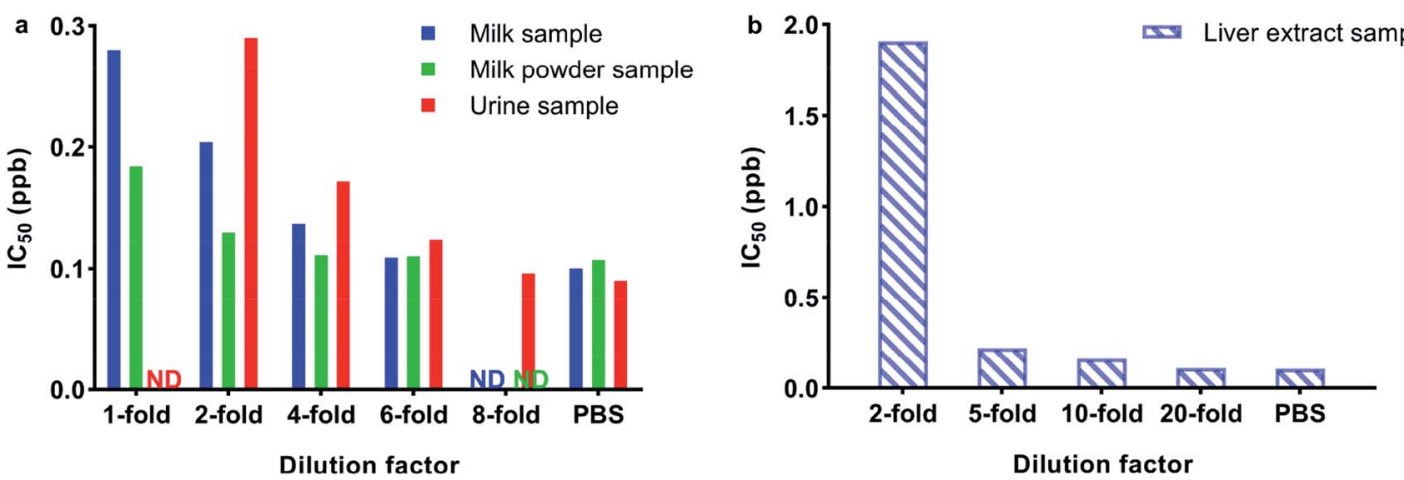

Fig. $5 \quad I_{50}$ values for (a) milk, skim milk powder, urine and (b) liver following different dilutions with PBS; ND: not detected.

CRs ranging from $40.6 \%$ to $128.8 \%$. The results indicated that the mAb obtained in this work was very effectively inhibited by zeranol, ZEA and the analogues, without any cross reactions (the CRs were all less than $0.1 \%$ ) with other mycotoxins or other growth promoters.

\subsection{Matrix effects}

The matrix effect is one of the challenges in immunoassay methods, which can cause an inaccurate result or even a falsepositive result. In some studies, sample pre-treatment procedures such as solid phase extraction and liquid-liquid extraction were often tedious and time consuming. However, for immunoassay methods, the preparation procedures of samples should be simplified, and a dilution method is more suitable. To evaluate the matrix effects, the standard curves obtained from the prepared samples were compared with the curve generated from PBS, as shown in Fig. 4 and 5. As shown in Fig. 4 and 5, all the $B /$ $B_{0}$ values and $\mathrm{IC}_{50}$ values in milk, skim milk powder, urine and liver samples were larger than that in PBS. The results indicated that the matrix effect existed in all cattle origin samples for this study, but it could be eliminated through the above sample pretreatment procedures. As shown in Fig. $4 \mathrm{a}$ and $5 \mathrm{a}$, take the standard curves and $\mathrm{IC}_{50}$ values in the milk sample as an example, the results indicated that after diluting 6-fold or more, there were no statistically significant differences in the $\mathrm{IC}_{50}$ values between the milk sample and PBS. For the milk powder sample and urine sample, they needed to be diluted 4-fold and 8fold respectively (Fig. 4b, c and 5a). For the liver extract sample, after diluting 20 -fold, the matrix effect could be removed, to ensure uniform experimental conditions, $4 \%$ methanol (v/v) was added to PBS (Fig. 4d and 5b). Meanwhile, similar results were obtained for muscle samples and kidney samples. Thus, the icELISA method can be used for the semiquantitative or quantitative detection of ZENs in milk, skim milk powder and urine after diluting the samples. As for the edible bovine tissues, such as muscle, liver and kidney, the pre-treatment procedures were similar to the above with some slight modifications. Firstly, the samples were extracted by methanol. Then, the extracted examples were analysed by the ic-ELISA method at 20-fold dilution. In practical applications, to ensure the concentrations of analytes are within the detection range by ic-ELISA, the sample dilution factor was required to be $>1$.

\subsection{Validation of the ic-ELISA method}

Zeranol was selected as the analyte to validate the ic-ELISA method. As shown in Table 3, the LOD values and LOQ values for zeranol in the cattle origin samples, including milk, skim milk powder, bovine urine, bovine liver, bovine muscle and bovine kidney, ranged from 0.016-0.027 ppb and 0.027$0.066 \mathrm{ppb}$, respectively. The RVs for the spiked samples were $79.2-104.2 \%$, and the CVs were less than $11.4 \%$. In addition, there was a good correlation $\left(R^{2}=0.9845\right)$ between the icELISA and HPLC-MS/MS results (Fig. 6), indicating that the proposed ic-ELISA was reliable for real applications. These results demonstrated that the developed ic-ELISA could meet the requirements of real-time monitoring zeranol and its analogues with broad specificity and high sensitivity in cattle origin samples.

\subsection{Evaluation of ELISA and other immunoassay methods}

In recent years, ELISA is the most emphatically developed and gradually commercialized immunoassay method for rapid detection of various analytes. There are indeed some developed

Table 3 LODs, LOQs, RVs, and CVs for zeranol in cattle origin samples

\begin{tabular}{lccllr}
\hline Sample & $\begin{array}{l}\text { LOD } \\
(\mathrm{ppb})\end{array}$ & $\begin{array}{l}\text { LOQ } \\
(\mathrm{ppb})\end{array}$ & $\begin{array}{l}\text { Spiked } \\
(\mathrm{ppb})\end{array}$ & $\begin{array}{l}\text { Mean RV } \\
(\%)\end{array}$ & $\begin{array}{r}\text { CV } \\
(\%)\end{array}$ \\
\hline Milk & \multirow{2}{*}{0.022} & 0.043 & 0.043 & 87.5 & 5.7 \\
& & & 0.086 & 92.6 & 6.8 \\
Skim milk & & & 0.215 & 94.3 & 7.1 \\
powder & 0.016 & 0.034 & 0.034 & 85.1 & 4.5 \\
& & & 0.068 & 95.2 & 4.7 \\
Urine & & & 0.171 & 89.0 & 9.3 \\
& 0.017 & 0.027 & 0.024 & 80.2 & 7.2 \\
& & & 0.054 & 90.3 & 1.8 \\
Liver & & & 0.137 & 104.2 & 8.6 \\
& 0.023 & 0.052 & 0.052 & 88.8 & 6.7 \\
Muscle & & & 0.104 & 85.4 & 10.1 \\
& & & 0.261 & 79.2 & 10.5 \\
& 0.026 & 0.062 & 0.062 & 84.2 & 7.6 \\
Kidney & & & 0.124 & 86.5 & 9.7 \\
& & & 0.310 & 87.2 & 10.2 \\
& 0.027 & 0.066 & 0.066 & 84.6 & 8.9 \\
& & & 0.131 & 87.6 & 11.4 \\
& & & 0.328 & 90.7 & 7.9
\end{tabular}




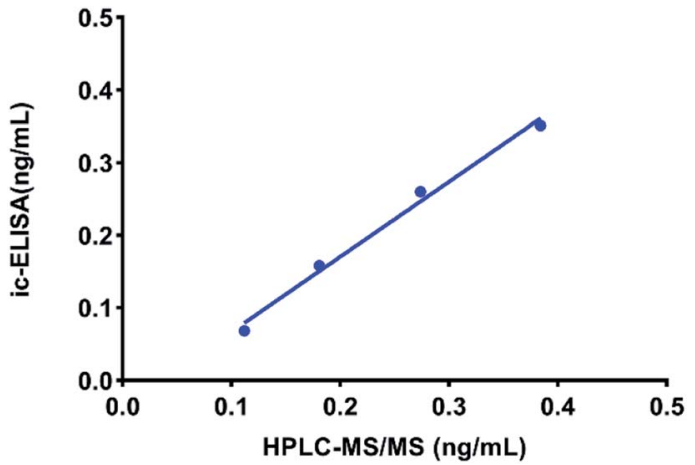

Fig. 6 Correlation between the analysis of zeranol in milk via ic-ELISA and HPLC-MS/MS methods.

methods for detecting zeranol, but none of them meet the requirements of detecting all ZENs with broad specificity, similar sensitivity and uniform affinity. Jiang et al. ${ }^{25}$ developed a micro-plate CLIEA that could just analyse zeranol and ZEA in bovine tissues, and both the methods developed by Yuan et al. ${ }^{22}$ and $\mathrm{Zhu}$ et al. ${ }^{19}$ have similar limitations. Even the commercialized ELISA kit did not perform as hoped, the Randox Food Diagnostics Zeranol ELISA test kit (ZR2421) could detect just half of six ZENs at one time and the sensitivity was lower than that of our developed ELISA method. Compared with these studies, the developed mAb in this study shows higher sensitivity and better cross-reactivity values, which successfully meet the requirements of broad-spectrum detection and more accurate results for detecting total levels of ZENs in one sample at one time.

\section{Conclusions}

In summary, we prepared a broad-spectrum mAb against zeranol and its five analogues, and then developed an ic-ELISA method for the determination of zeranol and its analogues in cattle origin samples. After simple sample preparation, the icELISA method could be used for the semiquantitative and quantitative detection of ZENs in bovine muscle, liver, kidney, urine, milk, and skim milk powder. The obtained detection limits were much lower than the permissible levels of zeranol and other ZENs, and excellent correlation $\left(R^{2}=0.9845\right)$ was achieved between ic-ELISA and HPLC-MS/MS, which indicated that ic-ELISA could reliably detect ZEN residues in various bovine samples. Therefore, the as-prepared method has been successfully applied in real samples and shows great potential to be used in the field of food safety.

\section{Ethical statement}

All animals were raised and treated according to the principles of the Institutional Animal Care and Use Committees of the Henan Academy of Agricultural Sciences and approved by the Animal Ethics Committee of Henan Academy of Agricultural Sciences (approval number SYXK 2014-0007).

\section{Conflicts of interest}

There are no conflicts to declare.

\section{Acknowledgements}

This work was supported by the China Postdoctoral Science Foundation Funded Project (2018M632778) and the Scientific and Technological Research Project of Henan Province (192102310513).

\section{References}

1 M. Stob, R. S. Baldwin, J. Tuite, F. N. Andrews and K. G. Gillette, Nature, 1962, 196, 1318.

2 T. W. Perry, M. Stob, D. A. Huber and R. C. Peterson, J. Anim. Sci., 1970, 31, 789-793.

3 G. D. Sharp and I. A. Dyer, J. Anim. Sci., 1971, 33, 865-871.

4 J. R. Roy, S. Chakraborty and T. R. Chakraborty, Med. Sci. Monit., 2009, 15, 137-145.

5 M. Metzler, E. Pfeiffer and A. A. Hildebrand, World Mycotoxin J., 2010, 3, 385-401.

6 H. Belhassen, I. Jiménez-Díaz, J. P. Arrebola, R. Ghali, H. Ghorbel, N. Olea and A. Hedili, Chemosphere, 2015, 128, $1-6$.

7 K. Tachampa, M. Takeda, S. Khamdang, R. Noshiro-Kofuji, M. Tsuda, S. Jariyawat, T. Fukutomi, S. Sophasan, N. Anzai and H. Endou, J. Pharmacol. Sci., 2008, 106, 435-443.

8 S. Liu and Y. C. Lin, Breast J., 2004, 10, 514-521.

9 W. M. Haschek and K. A. Voss, in Haschek and Rousseaux's Handbook of Toxicologic Pathology, 3rd edn, 2013, pp. 11871258.

10 B. Kabak, J. Sci. Food Agric., 2009, 89, 549-554.

11 Australian Pesticides and Veterinary Medicines Authority, Agricultural and Veterinary Chemicals Code (MRL Standard) Instrument 2019, 2019.

12 Government of Canada, List of Maximum Residue Limits (MRLs) for Veterinary Drugs in Foods, 2018.

13 European Commission, Off. J. Eur. Communities, 1996, 125, 3-9.

14 Ministry of Agriculture of the People's Republic of China, Announcement No. 235, 2002 of the Ministry of Agriculture of the People's Republic of China regarding to the maximum residue limits of veterinary drugs in animal foods, 2002.

15 L. C. Dickson, R. Costain, D. McKenzie, A. C. E. Fesser and J. D. MacNeil, J. Agric. Food Chem., 2009, 57, 6536-6542.

16 X. Sun, Q. Tang, X. Du, C. Xi, B. Tang, G. Wang and H. Zhao, Food Anal. Methods, 2017, 10, 3239-3246.

17 B. Socas-Rodríguez, J. González-Sálamo, J. Hernández-Borges and M. Á. R. Delgado, Electrophoresis, 2016, 37, 1359-1366.

18 S. N. Dixon, J. Vet. Pharmacol. Ther., 1980, 3, 177-181.

19 J. Zhu, X. Tao, S. Ding, J. Shen, Z. Wang, Y. Wang, F. Xu, X. Wu, T. Hu, A. Zhu and H. Jiang, Anal. Lett., 2012, 45, 2538-2548.

20 M. Regiart, M. A. Seia, G. A. Messina, F. A. Bertolino and J. Raba, Microchim. Acta, 2015, 182, 531-538. 
21 M. Tuomola, K. M. Cooper, S. Lahdenperä, G. A. Baxter, C. T. Elliott, D. G. Kennedy and T. Lövgren, Analyst, 2002, 127, 83-86.

22 Y. Liu, C. Zhang, X. Yu, Z. Zhang, X. Zhang, R. Liu, X. Liu and Z. Gong, J. Zhejiang Univ., Sci., B, 2007, 8, 900-905.
23 D. Thouvenot and R. F. Morfin, Appl. Environ. Microbiol., 1983, 45, 16-23.

24 G. Köhler and C. Milstein, Nature, 1975, 256, 495-497.

25 H. Jiang, W. Wang, J. Zhu, X. Tao, J. Li, X. Xia, K. Wen, F. Xu, Z. Wang, M. Chen, X. Li, X. Wu, S. Wang and S. Ding, Luminescence, 2014, 29, 393-400. 